\title{
POSITIVE INCREASING SOLUTIONS ON THE HALF-LINE TO SECOND ORDER NONLINEAR DELAY DIFFERENTIAL EQUATIONS
}

\author{
CH. G. PHILOS \\ Department of Mathematics, University of Ioannina, P. O. Box 1186, 45110 Ioannina, Greece \\ e-mail:cphilos@cc.uoi.gr
}

(Received 10 February, 2006; revised 15 March, 2007; accepted 31 March, 2007)

\begin{abstract}
Second order nonlinear delay differential equations with positive delays are considered, and sufficient conditions are given that guarantee the existence of positive increasing solutions on the half-line with first order derivatives tending to zero at infinity. The approach is elementary and is essentially based on an old idea which appeared in the author's paper Arch. Math. (Basel) 36 (1981), 168-178. The application of the result obtained to second order Emden-Fowler type differential equations with constant delays and, especially, to second order linear differential equations with constant delays, is also presented. Moreover, some (general or specific) examples demonstrating the applicability of the main result are given.
\end{abstract}

2000 Mathematics Subject Classification. 34K10, 34B18, 34B40

1. Introduction and statement of the main result. An important topic in the asymptotic theory of ordinary and delay differential equations is that of deriving sufficient conditions to ensure the existence of solutions with prescribed asymptotic behavior. Numerous articles have appeared in the literature on this topic; we choose to refer to $[\mathbf{3}, \mathbf{6}-\mathbf{1 0}, \mathbf{1 2}-\mathbf{1 8}, \mathbf{2 0}-\mathbf{3 0}]$ (and the references cited therein). In particular, it is of great interest to establish sufficient conditions for the existence of global solutions (i.e., of solutions on the whole given interval) with prescribed asymptotic behavior. As it concerns second order nonlinear ordinary or delay differential equations, the investigation of the existence of global solutions with prescribed asymptotic behavior is usually reduced to the study of the existence of solutions of boundary value problems on the half-line (see, for example, $[9,10,12,13,26-30]$ ). Boundary value problems on infinite intervals have many applications in physical problems (cf. [1]). The present article is concerned with the existence of positive solutions of a boundary value problem on the half-line to second order nonlinear delay differential equations. For the basic theory of delay differential equations, the reader is referred to the books $[\mathbf{4}, \mathbf{5}]$. Our work here is closely related to the work in the recent papers by Mavridis, the author and Tsamatos $[12,13]$ and, in a sense, to the work in the recent paper by Agarwal, the author and Tsamatos [2].

In this paper, we consider second order nonlinear delay differential equations with positive delays, and we establish sufficient conditions for the existence of positive increasing solutions on the half-line with first order derivatives tending to zero at infinity. The assumption that the delays are positive is essential to our approach, and hence our results cannot be applied to the corresponding second order nonlinear 
ordinary differential equations (see Section 4). An old idea that appeared in the author's paper [19] plays a crucial role in this paper. (Grains of this idea were presented in the paper by Lovelady [11].)

Consider the second order nonlinear delay differential equation

$$
x^{\prime \prime}(t)+f\left(t, x\left(t-T_{1}(t)\right), \ldots, x\left(t-T_{m}(t)\right)\right)=0,
$$

where $m$ is a positive integer, $f$ is a continuous real-valued function on the set $[0, \infty) \times \mathbb{R}^{m}$, and $T_{j}(j=1, \ldots, m)$ are positive continuous real-valued functions on the interval $[0, \infty)$ such that

$$
\lim _{t \rightarrow \infty}\left(t-T_{j}(t)\right)=\infty \quad(j=1, \ldots, m)
$$

Define

$$
\tau=-\min _{j=1, \ldots, m} \min _{t \geq 0}\left(t-T_{j}(t)\right)
$$

Clearly, $\tau$ is a positive real number.

Our interest will be concentrated on solutions of the delay differential equation $(1.1)$ on the whole interval $[0, \infty)$. By a solution on $[0, \infty)$ of $(1.1)$, we mean a continuous real-valued function $x$ defined on the interval $[-\tau, \infty)$ that is twice continuously differentiable on $[0, \infty)$ and satisfies $(1.1)$ for all $t \geq 0$.

Together with the delay differential equation (1.1), we specify an initial condition of the form

$$
x(t)=\phi(t) \text { for }-\tau \leq t \leq 0,
$$

where the initial function $\phi$ is a given continuous real-valued function on the interval $[-\tau, 0]$. Throughout the paper, it will be assumed that

$$
\phi(0)=0
$$

Moreover, along with (1.1), we associate the condition

$$
\lim _{t \rightarrow \infty} x^{\prime}(t)=0
$$

It must be noted that (1.3) implies that $\lim _{t \rightarrow \infty}[x(t) / t]=0$.

The delay differential equation (1.1) together with the conditions (1.2) and (1.3) constitute a boundary value problem (BVP, for short) on the half-line. A solution on $[0, \infty)$ of (1.1) satisfying (1.2) and (1.3) is said to be a solution of the boundary value problem (1.1)-(1.3) or, more briefly, a solution of the BVP (1.1)-(1.3).

The proposition given below provides a useful integral representation of the BVP (1.1)-(1.3), which will be used in proving the main result of the paper. This proposition has been established by Mavridis, the author and Tsamatos in [12] for a more general second order nonlinear delay boundary value problem, in which, however, the delays are assumed to be bounded. But, as it is easy to see, the restriction of the boundedness of the delays is not needed for the validity of the proposition. A similar proposition has also been used by Mavridis, the author and Tsamatos in the recent paper [13]. 
Proposition. A continuous real-valued function $x$ defined on the interval $[-\tau, \infty)$ is a solution of the BVP (1.1)-(1.3) if and only if it satisfies

$$
x(t)=\left\{\begin{array}{l}
\phi(t) \quad \text { for }-\tau \leq t \leq 0 \\
\int_{0}^{\infty} \min \{t, s\} f\left(s, x\left(s-T_{1}(s)\right), \ldots, x\left(s-T_{m}(s)\right)\right) d s \\
\quad=\int_{0}^{t} s f\left(s, x\left(s-T_{1}(s)\right), \ldots, x\left(s-T_{m}(s)\right)\right) d s \\
\quad+t \int_{t}^{\infty} f\left(s, x\left(s-T_{1}(s)\right), \ldots, x\left(s-T_{m}(s)\right)\right) d s \quad \text { for } t \geq 0 .
\end{array}\right.
$$

In the present paper, we are interested in studying the problem of the existence of solutions of the BVP (1.1)-(1.3) that are positive on $[-\tau, \infty)-\{0\}$. Therefore, in addition to the assumption that $\phi(0)=0$ posed previously, without mentioning it any further, it will be supposed that

$$
\phi(t)>0 \quad \text { for }-\tau \leq t<0 .
$$

The main result of this paper is the following theorem, which provides sufficient conditions for the BVP (1.1)-(1.3) to have at least one solution that is positive on $(0, \infty)$ and strictly increasing on $[0, \infty)$.

THEOREM. Suppose that the function $f$ is positive on $[0, \infty) \times(0, \infty)^{m}$; i.e.,

$$
f\left(t, y_{1}, \ldots, y_{m}\right)>0 \text { for all } t \geq 0 \text { and } y_{1}>0, \ldots, y_{m}>0 \text {. }
$$

Also, assume that, for each $t \geq 0$, the function $f(t, \cdot, \ldots, \cdot)$ is increasing on $[0, \infty)^{m}$ in the sense that $f\left(t, y_{1}, \ldots, y_{m}\right) \leq f\left(t, w_{1}, \ldots, w_{m}\right)$ for any $\left(y_{1}, \ldots, y_{m}\right),\left(w_{1}, \ldots, w_{m}\right)$ with $0 \leq y_{1} \leq w_{1}, \ldots, 0 \leq y_{m} \leq w_{m}$.

Let there exist a real number $c>0$ so that

$$
\int_{0}^{\infty} f\left(t, \rho_{1}(t), \ldots, \rho_{m}(t)\right) d t \leq c
$$

where, for each $j \in\{1, \ldots, m\}$, the function $\rho_{j}$ depends on $\phi, c$ and is defined by

$$
\rho_{j}(t)= \begin{cases}\phi\left(t-T_{j}(t)\right), & \text { if } 0 \leq t \leq T_{j}(t) \\ c\left(t-T_{j}(t)\right), & \text { if } t \geq T_{j}(t) .\end{cases}
$$

(Clearly, $\rho_{j}(j=1, \ldots, m)$ are nonnegative continuous real-valued functions on the interval $[0, \infty)$.) Then the BVP(1.1)-(1.3) has at least one solution $x$ such that

$$
0<x(t) \leq c t \text { for every } t>0
$$

and

$$
0<x^{\prime}(t) \leq c \text { for every } t \geq 0
$$

Note. Because of $x(0)=\phi(0)=0,(1.8)$ is a consequence of (1.9).

We notice here that, because of the continuity of $f$ on $[0, \infty) \times[0, \infty)^{m}$, the hypothesis that $f$ is positive on $[0, \infty) \times(0, \infty)^{m}$, i.e., that $(1.5)$ holds, implies that the function $f$ is nonnegative on $[0, \infty) \times[0, \infty)^{m}$, i.e.,

$$
f\left(t, y_{1}, \ldots, y_{m}\right) \geq 0 \text { for all } t \geq 0 \text { and } y_{1} \geq 0, \ldots, y_{m} \geq 0 \text {. }
$$


The proof of our theorem will be given in Section 2. Section 3 contains the application of the main result to second order Emden-Fowler type differential equations with constant delays, and, especially, to second order linear differential equations with constant delays. Also, some (general or specific) examples, which demonstrate the applicability of our theorem, will be presented in Section 3. The last section (Section 4) is devoted to a discussion.

2. Proof of the theorem. The following lemma provides useful information about the solutions of the BVP (1.1)-(1.3) that are nonnegative on the interval $(0, \infty)$. This lemma plays a crucial role in proving our theorem.

LEMMA. Suppose that the function $f$ is positive on $[0, \infty) \times(0, \infty)^{m}$; i.e., (1.5) holds. Let $x$ be a solution of the BVP (1.1)-(1.3) that is nonnegative on the interval $(0, \infty)$. Then $x$ is always positive on $(0, \infty)$; moreover, we have

$$
x^{\prime}(t)>0 \text { for every } t \geq 0
$$

and so $x$ is strictly increasing on $[0, \infty)$.

Note. Because of $x(0)=\phi(0)=0$, the positivity of $x$ on $(0, \infty)$ is a consequence of the fact that $x$ is strictly increasing on $[0, \infty)$.

Proof of the lemma. The proof will be accomplished by proving that $x^{\prime}$ is positive on the interval $[0, \infty)$ (and so $x$ is strictly increasing on $[0, \infty)$ ).

First of all, we observe that $x$ is nonnegative on the whole interval $[-\tau, \infty)$. Thus, we must have $x\left(t-T_{j}(t)\right) \geq 0$ for $t \geq 0(j=1, \ldots, m)$ and consequently, in view of (1.10),

$$
f\left(t, x\left(t-T_{1}(t)\right), \ldots, x\left(t-T_{m}(t)\right)\right) \geq 0 \quad \text { for every } t \geq 0 .
$$

Moreover, we see that, by our proposition, the solution $x$ satisfies (1.4). It follows immediately from (1.4) that

$$
x^{\prime}(t)=\int_{t}^{\infty} f\left(s, x\left(s-T_{1}(s)\right), \ldots, x\left(s-T_{m}(s)\right)\right) d s \quad \text { for all } t \geq 0 .
$$

Now, we shall show that $x^{\prime}(0)>0$. For this purpose, we apply (2.2) for $t=0$ to obtain

$$
x^{\prime}(0)=\int_{0}^{\infty} f\left(s, x\left(s-T_{1}(s)\right), \ldots, x\left(s-T_{m}(s)\right)\right) d s .
$$

Since $\quad-\tau \leq-T_{j}(0)<0 \quad(j=1, \ldots, m)$, we have $\quad x\left(-T_{j}(0)\right)=\phi\left(-T_{j}(0)\right)>0$, for $j=1, \ldots, m$ and so because of (1.5) we always have

$$
f\left(0, x\left(-T_{1}(0)\right), \ldots, x\left(-T_{m}(0)\right)\right)>0
$$

that is

$$
\left.f\left(t, x\left(t-T_{1}(t)\right), \ldots, x\left(t-T_{m}(t)\right)\right)\right|_{t=0}>0 .
$$

In view of (2.1) and the last inequality, it follows from (2.3) that $x^{\prime}(0)$ is necessarily positive. 
Next, we shall prove that $x^{\prime}$ is positive on the interval $(0, \infty)$. Assume, for the sake of contradiction, that $x^{\prime}$ is not always positive on $(0, \infty)$. Then, as $x^{\prime}(0)>0$, we see that $x^{\prime}$ must have zeros in the interval $(0, \infty)$. Let $t_{0}>0$ be the first zero of $x^{\prime}$ in $(0, \infty)$. That is, $x^{\prime}$ is positive on $\left[0, t_{0}\right)$, and $x^{\prime}\left(t_{0}\right)=0$. Thus, $x$ is strictly increasing on $\left[0, t_{0}\right)$ and $x$ in increasing on $\left[0, t_{0}\right]$. Hence, as $x(0)=\phi(0)=0$, we conclude that $x$ is always positive on the interval $\left(0, t_{0}\right]$. Furthermore, as $x^{\prime}\left(t_{0}\right)=0$, an application of (2.2) with $t=t_{0}$ gives

$$
\int_{t_{0}}^{\infty} f\left(s, x\left(s-T_{1}(s)\right), \ldots, x\left(s-T_{m}(s)\right)\right) d s=0,
$$

which, because of (2.1), yields

$$
f\left(t, x\left(t-T_{1}(t)\right), \ldots, x\left(t-T_{m}(t)\right)\right)=0 \quad \text { for every } t \geq t_{0} .
$$

By (2.4), it follows from (1.1) that $x^{\prime \prime}(t)=0$ for all $t \geq t_{0}$, which implies that $x^{\prime}$ is constant on $\left[t_{0}, \infty\right)$. Hence, since $x^{\prime}\left(t_{0}\right)=0$, we have $x^{\prime}(t)=0$ for every $t \geq t_{0}$. Consequently, $x$ is constant on $\left[t_{0}, \infty\right)$. Thus, as $x\left(t_{0}\right)>0$, we have $x(t)>0$ for any $t \geq t_{0}$. Hence, the solution $x$ is necessarily positive on the interval $(0, \infty)$. Now, by taking into account the assumption that $\lim _{t \rightarrow \infty}\left(t-T_{j}(t)\right)=\infty(j=1, \ldots, m)$, we can choose a point $t_{1}>0$ so that $t-T_{j}(t)>0$ for all $t \geq t_{1}(j=1, \ldots, m)$. Then, since $x$ is positive on $(0, \infty)$, we have $x\left(t-T_{j}(t)\right)>0$ for every $t \geq t_{1}(j=1, \ldots, m)$. Therefore, by virtue of (1.5), we obtain

$$
f\left(t, x\left(t-T_{1}(t)\right), \ldots, x\left(t-T_{m}(t)\right)\right)>0 \text { for all } t \geq t_{1},
$$

which contradicts (2.4).

The proof of the lemma is complete.

Now, we are in a position to proceed to the proof of our theorem.

Proof of the theorem. Let $X$ be the set of all continuous real-valued functions $x$ defined on the interval $[-\tau, \infty)$, that satisfy

$$
x(t)=\phi(t) \text { for }-\tau \leq t \leq 0
$$

and

$$
0 \leq x(t) \leq c t \text { for every } t>0 .
$$

Consider an arbitrary function $x$ in $X$. It follows from (2.5) and (2.6) that, for any $j \in\{1, \ldots, m\}$ and every $t \geq 0$,

$$
\begin{cases}0 \leq x\left(t-T_{j}(t)\right)=\phi\left(t-T_{j}(t)\right) & \text { if } 0 \leq t \leq T_{j}(t), \\ 0 \leq x\left(t-T_{j}(t)\right) \leq c\left(t-T_{j}(t)\right) & \text { if } t \geq T_{j}(t) .\end{cases}
$$

(Note that $x(0)=\phi(0)=0$.) In view of (1.7), we have

$$
0 \leq x\left(t-T_{j}(t)\right) \leq \rho_{j}(t) \quad \text { for every } t \geq 0 \quad(j=1, \ldots, m) .
$$

Thus, by using (1.10) as well as the hypothesis that, for each $t \geq 0$, the function $f(t, \cdot, \ldots, \cdot)$ is increasing on $[0, \infty)^{m}$, we find that

$$
0 \leq f\left(t, x\left(t-T_{1}(t)\right), \ldots, x\left(t-T_{m}(t)\right)\right) \leq f\left(t, \rho_{1}(t), \ldots, \rho_{m}(t)\right) \text { for all } t \geq 0 .
$$


Hence,

$$
0 \leq \int_{0}^{t} s f\left(s, x\left(s-T_{1}(s)\right), \ldots, x\left(s-T_{m}(s)\right)\right) d s \quad \text { for } t \geq 0 .
$$

Furthermore, we observe that the asssumption (1.6) guarantees, in particular, that

$$
\int_{0}^{\infty} f\left(t, \rho_{1}(t), \ldots, \rho_{m}(t)\right)<\infty
$$

and consequently, by virtue of (2.7), we must have

$$
0 \leq \int_{t}^{\infty} f\left(s, x\left(s-T_{1}(s)\right), \ldots, x\left(s-T_{m}(s)\right)\right) d s<\infty \quad \text { for } t \geq 0 .
$$

For any function $x$ in $X,(2.8)$ and (2.10) hold true and so we immediately see that the formula

$$
(M x)(t)=\left\{\begin{aligned}
\phi(t) & \text { for }-\tau \leq t \leq 0 \\
\int_{0}^{\infty} & \min \{t, s\} f\left(s, x\left(s-T_{1}(s)\right), \ldots, x\left(s-T_{m}(s)\right)\right) d s \\
= & \int_{0}^{t} s f\left(s, x\left(s-T_{1}(s)\right), \ldots, x\left(s-T_{m}(s)\right)\right) d s \\
\quad & +t \int_{t}^{\infty} f\left(s, x\left(s-T_{1}(s)\right), \ldots, x\left(s-T_{m}(s)\right)\right) d s \text { for } t \geq 0
\end{aligned}\right.
$$

makes sense for any function $x$ in $X$, and that this formula defines a mapping $M$ of $X$ into the set of all nonnegative continuous real-valued functions defined on the interval $[-\tau, \infty)$. We shall show that $M$ is a mapping of $X$ into itself; i.e., that $M X \subseteq X$. We observe that, for any function $x$ in $X$, we have $(M x)(t)=\phi(t)$ for $-\tau \leq t \leq 0$. Furthermore, let us consider an arbitrary function $x$ in $X$. By (2.8) and (2.10), we have $(M x)(t) \geq 0$ for every $t>0$. Moreover, by taking into account (2.7), we obtain, for $t \geq 0$,

$$
\begin{aligned}
(M x)^{\prime}(t) & =\int_{t}^{\infty} f\left(s, x\left(s-T_{1}(s)\right), \ldots, x\left(s-T_{m}(s)\right)\right) d s \\
& \leq \int_{t}^{\infty} f\left(s, \rho_{1}(s), \ldots, \rho_{m}(s)\right) d s \\
& \leq \int_{0}^{\infty} f\left(s, \rho_{1}(s), \ldots, \rho_{m}(s)\right) d s .
\end{aligned}
$$

Hence, by using the assumption (1.6), we find that

$$
(M x)^{\prime}(t) \leq c \text { for every } t \geq 0
$$

Since $(M x)(0)=\phi(0)=0$, it follows from (2.11) that, for each $t>0$,

$$
(M x)(t)=\int_{0}^{t}(M x)^{\prime}(s) d s \leq \int_{0}^{t} c d s=c t .
$$

That is, we always have $(M x)(t) \leq c t$ for every $t>0$. We have thus proved that, for any $x \in X, M x$ belongs to $X$; that is $M X \subseteq X$.

Now, let us consider two arbitrary functions $x$ and $\tilde{x}$ in $X$ with $x \leq \tilde{x}$; i.e., with $x(t) \leq \widetilde{x}(t)$ for $t \geq-\tau$. Then, by the definition of $X$, for each $j \in\{1, \ldots, m\}$ and every 
$t \geq 0$, we have

$$
\left\{\begin{array}{l}
0 \leq x\left(t-T_{j}(t)\right)=\phi\left(t-T_{j}(t)\right)=\tilde{x}\left(t-T_{j}(t)\right) \quad \text { if } 0 \leq t \leq T_{j}(t) \\
0 \leq x\left(t-T_{j}(t)\right) \leq \tilde{x}\left(t-T_{j}(t)\right) \quad \text { if } t \geq T_{j}(t)
\end{array}\right.
$$

Thus, by taking into account the hypothesis that, for each $t \geq 0$, the function $f(t, \cdot, \ldots, \cdot)$ is increasing on $[0, \infty)^{m}$, we get

$$
f\left(t, x\left(t-T_{1}(t)\right), \ldots, x\left(t-T_{m}(t)\right)\right) \leq f\left(t, \widetilde{x}\left(t-T_{1}(t)\right), \ldots, \widetilde{x}\left(t-T_{m}(t)\right)\right) \text { for } t \geq 0 .
$$

Hence, we see that

$$
\begin{cases}(M x)(t)=(M \widetilde{x})(t) & \text { for }-\tau \leq t \leq 0 \\ (M x)(t) \leq(M \widetilde{x})(t) & \text { for } t \geq 0\end{cases}
$$

and consequently $(M x)(t) \leq(M \tilde{x})(t)$, for every $t \geq-\tau$; i.e., $M x \leq M \tilde{x}$. We have thus proved that the mapping $M$ is increasing with respect to the usual pointwise ordering in $X$.

Next, we define

$$
x_{0}(t)=\left\{\begin{array}{l}
\phi(t) \text { for }-\tau \leq t \leq 0 \\
c t \text { for } t \geq 0
\end{array}\right.
$$

and

$$
x_{v+1}=M x_{v} \quad(v=0,1, \ldots) .
$$

As $M$ is an increasing mapping of $X$ into itself, it is not difficult to see that $\left(x_{v}\right)_{v=0,1, \ldots}$ is a decreasing sequence of functions in $X$. Set

$$
x=\lim _{\nu \rightarrow \infty} x_{v} \text { pointwise on }[-\tau, \infty) .
$$

By (2.7), we have for $t \geq 0$

$$
0 \leq f\left(t, x_{v}\left(t-T_{1}(t)\right), \ldots, x_{v}\left(t-T_{m}(t)\right)\right) \leq f\left(t, \rho_{1}(t), \ldots, \rho_{m}(t)\right)
$$

for all nonnegative integers $v$. Hence, because of (2.9), we can apply the Lebesgue dominated convergence theorem to obtain, for $t \geq 0$

$$
\begin{gathered}
\lim _{\nu \rightarrow \infty} \int_{0}^{\infty} \min \{t, s\} f\left(s, x_{v}\left(s-T_{1}(s)\right), \ldots, x_{v}\left(s-T_{m}(s)\right)\right) d s \\
\quad=\int_{0}^{\infty} \min \{t, s\} f\left(s, x\left(s-T_{1}(s)\right), \ldots, x\left(s-T_{m}(s)\right)\right) d s
\end{gathered}
$$

Thus, we conclude that

$$
\lim _{\nu \rightarrow \infty}\left(M x_{v}\right)(t)=(M x)(t), \quad \text { for every } t \geq-\tau .
$$

Consequently, we have

$$
x(t)=\lim _{\nu \rightarrow \infty} x_{\nu+1}(t)=\lim _{\nu \rightarrow \infty}\left(M x_{\nu}\right)(t)=(M x)(t) \quad \text { for } t \geq-\tau
$$


and so $x=M x$, i.e., $x$ satisfies (1.4). Therefore, by our proposition, $x$ is a solution of the BVP (1.1)-(1.3). As $x \in X$, the solution $x$ satisfies $0 \leq x(t) \leq c t$ for every $t>0$. Moreover, since $x=M x$, it follows from (2.11) that $x^{\prime}(t) \leq c$ for every $t \geq 0$. Finally, as $x$ is nonnegative on the interval $(0, \infty)$, our lemma guarantees that $x$ is always positive on $(0, \infty)$ and such that $x^{\prime}(t)>0$ for every $t \geq 0$. Hence the solution $x$ satisfies $(1.8)$ and (1.9).

The proof of the theorem is complete.

3. Applications and examples. Consider the second order Emden-Fowler type delay differential equation

$$
x^{\prime \prime}(t)+\sum_{j=1}^{m} p_{j}(t)\left|x\left(t-\tau_{j}\right)\right|^{\gamma_{j}} \operatorname{sgn} x\left(t-\tau_{j}\right)=0
$$

and, especially, the second order linear delay differential equation

$$
x^{\prime \prime}(t)+\sum_{j=1}^{m} p_{j}(t) x\left(t-\tau_{j}\right)=0,
$$

where $m$ is a positive integer, $p_{j}(j=1, \ldots, m)$ are nonnegative continuous real-valued functions on the interval $[0, \infty), \tau_{j}(j=1, \ldots, m)$ are positive real constants, and $\gamma_{j}$ $(j=1, \ldots, m)$ are positive real numbers. It will be supposed that

$$
\sum_{j=1}^{m} p_{j}(t)>0 \quad \text { for all } t \geq 0 .
$$

We notice that, as $p_{j}(j=1, \ldots, m)$ are nonnegative on $[0, \infty)$, the last hypothesis means exactly that, for each $t \geq 0$, there exists at least one index $j \in\{1, \ldots, m\}$ so that $p_{j}(t)>0$.

Let us define the positive real number $\tau$ by

$$
\tau=\max _{j=1, \ldots, m} \tau_{j}
$$

We are interested in solutions of the delay differential equation (3.1) (and, especially, of (3.2)) on the whole interval $[0, \infty)$. A solution on $[0, \infty)$ of $(3.1)$ (respectively, of (3.2)) is a continuous real-valued function $x$ defined on the interval $[-\tau, \infty)$, which is twice continuously differentiable on $[0, \infty)$ and satisfies (3.1) (respectively, (3.2)) for all $t \geq 0$. The initial condition (1.2) as well as the condition (1.3) are associated with (3.1) (and, especially, with (3.2)). Hence, we have the BVP (3.1), (1.2) and (1.3) (and, especially, the BVP (3.2), (1.2) and (1.3)).

By applying our theorem to the particular case of the BVP (3.1), (1.2) and (1.3), we are led to the following corollary.

COROLLARY 1. Let there exist a real number $c>0$ so that

$$
\sum_{j=1}^{m} \int_{0}^{\tau_{j}}\left[\phi\left(t-\tau_{j}\right)\right]^{\gamma_{j}} p_{j}(t) d t+\sum_{j=1}^{m} c^{\gamma_{j}} \int_{\tau_{j}}^{\infty}\left(t-\tau_{j}\right)^{\gamma_{j}} p_{j}(t) d t \leq c .
$$


Then the BVP (3.1), (1.2) and (1.3) has at least one solution $x$ such that (1.8) and (1.9) hold.

The linear delay differential equation (3.2) can be obtained (as a special case) from (3.1) by taking $\gamma_{j}=1$ for $j=1, \ldots, m$. In the special case of the BVP (3.2), (1.2) and (1.3), the hypothesis in Corollary 1 becomes

$$
\sum_{j=1}^{m} \int_{0}^{\tau_{j}} \phi\left(t-\tau_{j}\right) p_{j}(t) d t+c \sum_{j=1}^{m} \int_{\tau_{j}}^{\infty}\left(t-\tau_{j}\right) p_{j}(t) d t \leq c
$$

i.e.,

$$
\sum_{j=1}^{m} \int_{0}^{\tau_{j}} \phi\left(t-\tau_{j}\right) p_{j}(t) d t \leq c\left[1-\sum_{j=1}^{m} \int_{\tau_{j}}^{\infty}\left(t-\tau_{j}\right) p_{j}(t) d t\right]
$$

Hence, we can immediately arrive at the next corollary.

COROllary 2. Let the condition

$$
\sum_{j=1}^{m} \int_{\tau_{j}}^{\infty}\left(t-\tau_{j}\right) p_{j}(t) d t<1
$$

be satisfied, and set

$$
c=\frac{\sum_{j=1}^{m} \int_{0}^{\tau_{j}} \phi\left(t-\tau_{j}\right) p_{j}(t) d t}{1-\sum_{j=1}^{m} \int_{\tau_{j}}^{\infty}\left(t-\tau_{j}\right) p_{j}(t) d t}
$$

(Clearly, $c$ is a positive real number.) Then the BVP (3.2), (1.2) and (1.3) has at least one solution $x$ such that (1.8) and (1.9) hold.

Now, in order to present some examples demonstrating the applicability of our theorem, we shall concentrate on Emden-Fowler type differential equations (and, especially, linear differential equations) with one constant delay.

Let us consider the Emden-Fowler type delay differential equation

$$
x^{\prime \prime}(t)+p(t)|x(t-\tau)|^{\gamma} \operatorname{sgn} x(t-\tau)=0
$$

and, especially, the linear delay differential equation

$$
x^{\prime \prime}(t)+p(t) x(t-\tau)=0
$$

where $p$ is a positive continuous real-valued function on the interval $[0, \infty), \tau$ is a positive real constant, and $\gamma$ is a positive real number.

In the particular case of the BVP (3.3), (1.2) and (1.3), Corollary 1 is formulated as follows.

Let there exist a real number $c>0$ so that

$$
\int_{0}^{\tau}[\phi(t-\tau)]^{\gamma} p(t) d t+c^{\gamma} \int_{\tau}^{\infty}(t-\tau)^{\gamma} p(t) d t \leq c .
$$


Then the BVP (3.3), (1.2) and (1.3) has at least one solution $x$ such that (1.8) and (1.9) hold.

Moreover, as it concerns the special case of the BVP (3.4), (1.2) and (1.3), Corollary 2 takes the following form.

Let the condition

$$
\int_{\tau}^{\infty}(t-\tau) p(t) d t<1
$$

be satisfied, and set

$$
c=\frac{\int_{0}^{\tau} \phi(t-\tau) p(t) d t}{1-\int_{\tau}^{\infty}(t-\tau) p(t) d t} .
$$

Clearly, $c$ is a positive real number. Then the BVP (3.4), (1.2) and (1.3) has at least one solution $x$ such that (1.8) and (1.9) hold.

EXAMPLE 1. Consider the differential equation (3.3) with $\gamma=\frac{1}{2}$; i.e., the sublinear delay differential equation

$$
x^{\prime \prime}(t)+p(t)|x(t-\tau)|^{1 / 2} \operatorname{sgn} x(t-\tau)=0 .
$$

As it concerns the BVP (3.8), (1.2) and (1.3), condition (3.5) becomes

$$
\int_{0}^{\tau}[\phi(t-\tau)]^{1 / 2} p(t) d t+c^{1 / 2} \int_{\tau}^{\infty}(t-\tau)^{1 / 2} p(t) d t \leq c ;
$$

namely

$$
c-\left[\int_{\tau}^{\infty}(t-\tau)^{1 / 2} p(t) d t\right] c^{1 / 2}-\int_{0}^{\tau}[\phi(t-\tau)]^{1 / 2} p(t) d t \geq 0 .
$$

By taking $c^{1 / 2}=C$, the last inequality is written as

$$
C^{2}-\left[\int_{\tau}^{\infty}(t-\tau)^{1 / 2} p(t) d t\right] C-\int_{0}^{\tau}[\phi(t-\tau)]^{1 / 2} p(t) d t \geq 0 .
$$

Let us consider the quadratic equation

$$
\Omega(\omega) \equiv \omega^{2}-\left[\int_{\tau}^{\infty}(t-\tau)^{1 / 2} p(t) d t\right] \omega-\int_{0}^{\tau}[\phi(t-\tau)]^{1 / 2} p(t) d t=0
$$

in the complex plane, and let $\Delta$ be its discriminant; i.e.,

$$
\Delta=\left[\int_{\tau}^{\infty}(t-\tau)^{1 / 2} p(t) d t\right]^{2}+4 \int_{0}^{\tau}[\phi(t-\tau)]^{1 / 2} p(t) d t .
$$

We observe that $\Delta>0$. Hence, the equation $\Omega(\omega)=0$ has two distinct real roots $\omega_{1}$ and $\omega_{2}$ given by

$$
\omega_{1}=\frac{1}{2} \int_{\tau}^{\infty}(t-\tau)^{1 / 2} p(t) d t-\sqrt{\left[\frac{1}{2} \int_{\tau}^{\infty}(t-\tau)^{1 / 2} p(t) d t\right]^{2}+\int_{0}^{\tau}[\phi(t-\tau)]^{1 / 2} p(t) d t}
$$


and

$$
\omega_{2}=\frac{1}{2} \int_{\tau}^{\infty}(t-\tau)^{1 / 2} p(t) d t+\sqrt{\left[\frac{1}{2} \int_{\tau}^{\infty}(t-\tau)^{1 / 2} p(t) d t\right]^{2}+\int_{0}^{\tau}[\phi(t-\tau)]^{1 / 2} p(t) d t}
$$

We immediately see that $\omega_{1}<0<\omega_{2}$. For each real number $\omega$, we have $\Omega(\omega) \geq 0$ if and only if either $\omega \leq \omega_{1}$ or $\omega \geq \omega_{2}$. Hence, (3.10) is satisfied with $C>0$ if and only if $C \geq \omega_{2}$. Consequently, (3.9) holds with $c>0$ if and only if $c \geq \omega_{2}^{2}$. Thus, we conclude that (3.9) is valid (as an equality) for

$$
c=\left\{\frac{1}{2} \int_{\tau}^{\infty}(t-\tau)^{1 / 2} p(t) d t+\sqrt{\left[\frac{1}{2} \int_{\tau}^{\infty}(t-\tau)^{1 / 2} p(t) d t\right]^{2}+\int_{0}^{\tau}[\phi(t-\tau)]^{1 / 2} p(t) d t}\right\}^{2} .
$$

In particular, we obtain the following result.

Let $c>0$ be the real number given by (3.11). Then the BVP (3.8), (1.2) and (1.3) has at least one solution $x$ such that (1.8) and (1.9) hold.

Now, we choose $\tau=1, \phi(t)=-t$ for $-1 \leq t \leq 0$, and

$$
p(t)=e^{-|t-1|^{1 / 2}} \quad \text { for } t \geq 0 .
$$

Then we can easily show that

$$
\int_{\tau}^{\infty}(t-\tau)^{1 / 2} p(t) d t=4, \quad \int_{0}^{\tau}[\phi(t-\tau)]^{1 / 2} p(t) d t=4-\frac{10}{e}
$$

and consequently (3.11) becomes

$$
c=\left(2+\sqrt{8-\frac{10}{e}}\right)^{2}
$$

Hence, we arrive at the following result. The boundary value problem

$$
\left\{\begin{array}{l}
x^{\prime \prime}(t)+e^{-|t-1|^{1 / 2}}|x(t-1)|^{1 / 2} \operatorname{sgn} x(t-1)=0 \\
x(t)=-t \text { for }-1 \leq t \leq 0, \quad \lim _{t \rightarrow \infty} x^{\prime}(t)=0
\end{array}\right.
$$

has at least one solution $x$ such that (1.8) and (1.9) hold, where the positive real number $c$ is given by (3.12).

EXAMPLE 2. Let us consider the case of the differential equation (3.3) with $\gamma=2$; i.e., the case of the superlinear delay differential equation

$$
x^{\prime \prime}(t)+p(t)[x(t-\tau)]^{2} \operatorname{sgn} x(t-\tau)=0 .
$$

In the case of the BVP (3.13), (1.2) and (1.3), condition (3.5) is written as

$$
\int_{0}^{\tau}[\phi(t-\tau)]^{2} p(t) d t+c^{2} \int_{\tau}^{\infty}(t-\tau)^{2} p(t) d t \leq c
$$


or

$$
\left[\int_{\tau}^{\infty}(t-\tau)^{2} p(t) d t\right] c^{2}-c+\int_{0}^{\tau}[\phi(t-\tau)]^{2} p(t) d t \leq 0 .
$$

Consider the quadratic equation

$$
\Omega(\omega) \equiv\left[\int_{\tau}^{\infty}(t-\tau)^{2} p(t) d t\right] \omega^{2}-\omega+\int_{0}^{\tau}[\phi(t-\tau)]^{2} p(t) d t=0,
$$

in the complex plane, and denote by $\Delta$ its discriminant; i.e.,

$$
\Delta=1-4\left[\int_{\tau}^{\infty}(t-\tau)^{2} p(t) d t\right] \int_{0}^{\tau}[\phi(t-\tau)]^{2} p(t) d t .
$$

If $\Delta<0$, then we always have $\Omega(\omega)>0$ for all real numbers $\omega$, and so there is no real number $c>0$ such that (3.14) holds. Suppose that $\Delta=0$. Then the equation $\Omega(\omega)=0$ has exactly one (double) real root $\omega_{0}$ given by

$$
\omega_{0}=\frac{1}{2 \int_{\tau}^{\infty}(t-\tau)^{2} p(t) d t}
$$

which is obviously positive. For any real number $\omega$, we have $\Omega(\omega) \leq 0$ if and only if $\Omega(\omega)=0$; i.e., if and only if $\omega=\omega_{0}$. Consequently, (3.14) is satisfied with $c>0$ if and only if $c=\omega_{0}$. (For $c=\omega_{0},(3.14)$ is fulfilled as an equality.) Next, let us assume that $\Delta>0$. Then the equation $\Omega(\omega)=0$ has the real roots

$$
\omega_{1}=\frac{1-\sqrt{1-4\left[\int_{\tau}^{\infty}(t-\tau)^{2} p(t) d t\right] \int_{0}^{\tau}[\phi(t-\tau)]^{2} p(t) d t}}{2 \int_{\tau}^{\infty}(t-\tau)^{2} p(t) d t}
$$

and

$$
\omega_{2}=\frac{1+\sqrt{1-4\left[\int_{\tau}^{\infty}(t-\tau)^{2} p(t) d t\right] \int_{0}^{\tau}[\phi(t-\tau)]^{2} p(t) d t}}{2 \int_{\tau}^{\infty}(t-\tau)^{2} p(t) d t}
$$

with $0<\omega_{1}<\omega_{2}$. For each real number $\omega$, we have $\Omega(\omega) \leq 0$ if and only if $\omega_{1} \leq \omega \leq$ $\omega_{2}$. Thus, (3.14) holds with $c>0$ if and only if $\omega_{1} \leq c \leq \omega_{2}$. In particular, for $c=\omega_{1}$, (3.14) is satisfied (as an equality). After the above analysis, we conclude that, if

$$
\left[\int_{\tau}^{\infty}(t-\tau)^{2} p(t) d t\right] \int_{0}^{\tau}[\phi(t-\tau)]^{2} p(t) d t \leq \frac{1}{4}
$$

then (3.14) is valid (as an equality) for

$$
c=\frac{1-\sqrt{1-4\left[\int_{\tau}^{\infty}(t-\tau)^{2} p(t) d t\right] \int_{0}^{\tau}[\phi(t-\tau)]^{2} p(t) d t}}{2 \int_{\tau}^{\infty}(t-\tau)^{2} p(t) d t} .
$$

Hence, we are led to the next result.

Assume that (3.15) is satisfied, and let $c>0$ be the real number given by (3.16). Then the BVP (3.13), (1.2) and (1.3) has at least one solution $x$ such that (1.8) and (1.9) hold. 
Now, take $\tau=1, \phi(t)=-t$ for $-1 \leq t \leq 0$, and

$$
p(t)=\frac{3}{(t+1)^{4}} \quad \text { for } t \geq 0 .
$$

Then it is easy to find that

$$
\int_{\tau}^{\infty}(t-\tau)^{2} p(t) d t=\frac{1}{2} \quad \text { and } \quad \int_{0}^{\tau}[\phi(t-\tau)]^{2} p(t) d t=\frac{1}{2} .
$$

Hence, (3.15) is satisfied (as an equality) and (3.16) becomes $c=1$. Thus, we derive the following result. The boundary value problem

$$
\left\{\begin{array}{l}
x^{\prime \prime}(t)+\frac{3}{(t+1)^{4}}[x(t-1)]^{2} \operatorname{sgn} x(t-1)=0, \\
x(t)=-t \text { for }-1 \leq t \leq 0, \quad \lim _{t \rightarrow \infty} x^{\prime}(t)=0
\end{array}\right.
$$

has at least one solution $x$ such that (1.8) and (1.9) hold, with $c=1$.

Next, set $\tau=1, \phi(t)=-t$ for $-1 \leq t \leq 0$, and

$$
p(t)=\frac{3 \sqrt{3}}{2(t+1)^{4}} \quad \text { for } t \geq 0 .
$$

In this case, we find that

$$
\int_{\tau}^{\infty}(t-\tau)^{2} p(t) d t=\frac{\sqrt{3}}{4} \quad \text { and } \quad \int_{0}^{\tau}[\phi(t-\tau)]^{2} p(t) d t=\frac{\sqrt{3}}{4},
$$

and consequently (3.15) is fulfilled (as a strict inequality) and (3.16) gives $c=\frac{1}{\sqrt{3}}$. Thus, we arrive at the next result: The boundary value problem

$$
\left\{\begin{array}{l}
x^{\prime \prime}(t)+\frac{3 \sqrt{3}}{2(t+1)^{4}}[x(t-1)]^{2} \operatorname{sgn} x(t-1)=0 \\
x(t)=-t \text { for }-1 \leq t \leq 0, \quad \lim _{t \rightarrow \infty} x^{\prime}(t)=0
\end{array}\right.
$$

has at least one solution $x$ such that (1.8) and (1.9) hold, with $c=\frac{1}{\sqrt{3}}$.

EXAMPLE 3. Consider the linear delay differential equation (3.4) with $\tau=1$ and

$$
p(t)=\frac{2}{(t+1)^{3}} \quad \text { for } t \geq 0 .
$$

Take $\phi(t)=-t$ for $-1 \leq t \leq 0$. It is a matter of elementary calculation to show that

$$
\int_{\tau}^{\infty}(t-\tau) p(t) d t=\frac{1}{2} \quad \text { and } \quad \int_{0}^{\tau} \phi(t-\tau) p(t) d t=\frac{1}{2} .
$$

Hence, we see that (3.6) is satisfied and that (3.7) becomes $c=1$. Thus the following result is true. The boundary value problem

$$
\left\{\begin{array}{l}
x^{\prime \prime}(t)+\frac{2}{(t+1)^{3}} x(t-1)=0 \\
x(t)=-t \text { for }-1 \leq t \leq 0, \quad \lim _{t \rightarrow \infty} x^{\prime}(t)=0
\end{array}\right.
$$

has at least one solution $x$ such that (1.8) and (1.9) hold, with $c=1$. 
4. Discussion. As it is immediately seen, our lemma plays a crucial role in proving our theorem; i.e., the main result of the paper. Moreover, after a careful reading of the proof of our lemma, one may easily verify that the proof of this lemma is essentially based on the use of the hypothesis that the initial function $\phi$ is positive on the interval $[-\tau, 0)$ (as well as on the assumption that the function $f$ is positive on $[0, \infty) \times(0, \infty)^{m}$; i.e., that (1.5) holds). This hypothesis is fundamental, because of the fact that $\tau>0$; i.e., of the fact that the delays $T_{j}(j=1, \ldots, m)$ are positive on the interval $[0, \infty)$. It is clear that we cannot have such an hypothesis in the case of the second order nonlinear ordinary differential equations, and so our lemma (and, consequently, our theorem) cannot be applied to the corresponding ordinary boundary value problem. More precisely, let us consider the second order nonlinear delay differential equation

$$
x^{\prime \prime}(t)+f_{0}(t, x(t-\tau))=0,
$$

where $f_{0}$ is a continuous real-valued fucntion on $[0, \infty) \times \mathbb{R}$ that is positive on $[0, \infty) \times$ $(0, \infty)$ and $\tau$ is a positive real constant. For $\tau=0$, equation (4.1) reduces to the second order nonlinear ordinary differential equation

$$
x^{\prime \prime}(t)+f_{0}(t, x(t))=0,
$$

and the initial condition (1.2) becomes

$$
x(0)=0 .
$$

That is, when $\tau=0$, the BVP (4.1), (1.2), (1.3) reduces to the BVP (4.2), (4.3), (1.3). Our lemma and our theorem are applicable to the delay BVP (4.1), (1.2), (1.3), but these results cannot be applied to the ordinary BVP (4.2), (4.3), (1.3).

In Section 3 we have given examples of delay boundary value problems of the form (4.1), (1.2), (1.3) in which our theorem applies. But, it is difficult to verify that the corresponding ordinary boundary value problems of the form (4.2), (4.3), (1.3) have no positive increasing solutions, since in order to show this fact one has to solve explicitly the ordinary boundary value problems.

Finally, we notice that our theorem establishes sufficient conditions for the existence of at least one solution $x$ of the BVP (1.1)-(1.3), that is positive on $(0, \infty)$ and strictly increasing on $[0, \infty)$. It is remarkable that our lemma guarantees that any solution $x$ of the BVP (1.1)-(1.3) that is nonnegative on $(0, \infty)$, has the same behaviour; i.e., it is always positive on $(0, \infty)$ and strictly increasing on $[0, \infty)$.

\section{REFERENCES}

1. R. P. Agarwal and D. O'Regan, Infinite interval problems for differential, difference and integral equations (Kluwer Academic Publishers, Dordrecht, 2001).

2. R. P. Agarwal, Ch. G. Philos and P. Ch. Tsamatos, Global solutions of a singular initial value problem to second order nonlinear delay differential equations, Math. Comput. Modelling 43 (2006), 854-869.

3. A. Constantin, On the existence of positive solutions of second order differential equations, Ann. Mat. Pura Appl. 184 (2005), 131-138.

4. O. Diekmann, S. A. van Gils, S. M. Verduyn Lunel and H.-O. Walther, Delay equations: Functional-, Complex-, and Nonlinear Analysis (Springer-Verlag, 1995).

5. J. K. Hale and S. M. Verduyn Lunel, Introduction to functional differential equations (Springer-Verlag, 1993). 
6. T. Kusano and W. F. Trench, Existence of global solutions with prescribed asymptotic behavior for nonlinear ordinary differential equations, Ann. Mat. Pura Appl. 142 (1985), 381392.

7. T. Kusano and W. F. Trench, Global existence theorems for solutions of nonlinear differential equations with prescribed asymptotic behaviour, J. London Math. Soc. (2) 31 (1985), $478-486$.

8. O. Lipovan, On the asymptotic behaviour of the solutions to a class of second order nonlinear differential equations, Glasgow Math. J. 45 (2003), 179-187.

9. Y. Liu, Existence and unboundedness of positive solutions for singular boundary value problems on the half-line, Appl. Math. Comput. 144 (2003), 543-556.

10. Y. Liu, Boundary value problems on the half-line for functional differential equations with infinite delay in a Banach space, Nonlinear Anal. 52 (2003), 1695-1708.

11. D. Lovelady, Positive bounded solutions for a class of linear delay differential equations, Hiroshima Math. J. 6 (1976), 451-456.

12. K. G. Mavridis, Ch. G. Philos and P. Ch. Tsamatos, Existence of solutions of a boundary value problem on the half-line to second order nonlinear delay differential equations, Arch. Math. (Basel) 86 (2006), 163-175.

13. K. G. Mavridis, Ch. G. Philos and P. Ch. Tsamatos, Multiple positive solutions for a second order delay boundary value problem on the half-line, Ann. Polon. Math. 88 (2006), $173-191$.

14. O. Mustafa, On the existence of solutions with prescribed asymptotic behaviour for perturbed nonlinear differential equations of second order, Glasgow Math. J. 47 (2005), 177185.

15. O. Mustafa and Y. V. Rogovchenko, Global existence of solutions with prescribed asymptotic behavior for second-order nonlinear differential equations, Nonlinear Anal. 51 (2002), 339-368.

16. O. Mustafa and Y. V. Rogovchenko, Global existence and asymptotic behavior of solutions of nonlinear differential equations, Funkcial. Ekvac. 47 (2004), 167-186.

17. O. Mustafa and Y. V. Rogovchenko, Asymptotic integration of nonlinear differential equations, Nonlinear Anal. 63 (2005), e2135-e2143.

18. O. Mustafa and Y. V. Rogovchenko, Asymptotic integration of a class of nonlinear differential equations, Appl. Math. Lett. 19 (2006), 849-853.

19. Ch. G. Philos, On the existence of nonoscillatory solutions tending to zero at $\infty$ for differential equations with positive delays, Arch. Math. (Basel) 36 (1981), 168-178.

20. Ch. G. Philos, Asymptotic behaviour of a class of nonoscillatory solutions of differential equations with deviating arguments, Math. Slovaca 33 (1983), 409-428.

21. Ch. G. Philos, I. K. Purnaras and P. Ch. Tsamatos, Asymptotic to polynomials solutions for nonlinear differential equations, Nonlinear Anal. 59 (2004), 1157-1179.

22. Ch. G. Philos, I. K. Purnaras and P. Ch. Tsamatos, Global solutions approaching lines at infinity to second order nonlinear delay differential equations, Funkcial. Ekvac., to appear.

23. Ch. G. Philos, Y. G. Sficas and V. A. Staikos, Some results on the asymptotic behavior of nonoscillatory solutions of differential equations with deviating arguments, J. Austral. Math. Soc. Series A 32 (1982), 295-317.

24. Ch. G. Philos and V. A. Staikos, A basic asymptotic criterion for differential equations with deviating arguments and its applications to the nonoscillation of linear ordinary equations, Nonlinear Anal. 6 (1982), 1095-1113.

25. Ch. G. Philos and P. Ch. Tsamatos, Solutions approaching polynomials at infinity to nonlinear ordinary differential equations, Electron. J. Differential Equations 2005 (2005), No. 79, pp. 1-25.

26. Z. Wei and S. Chen, Positive solution of singular boundary value problems on a half-line, Acta Math. Appl. Sinica (English Ser.) 21 (2005), 553-564.

27. B. Yan, Multiple unbounded solutions of boundary value problems for second-order differential equations on the half-line, Nonlinear Anal. 51 (2002), 1031-1044.

28. B. Yan and Y. Liu, Unbounded solutions of the singular boundary value problems for second order differential equations on the half-line, Appl. Math. Comput. 147 (2004), 629-644.

29. Z. Yin, Monotone positive solutions of second-order nonlinear differential equations, Nonlinear Anal. 54 (2003), 391-403.

30. Z. Zhao, Positive solutions of nonlinear second order ordinary differential equations, Proc. Amer. Math. Soc. 121 (1994), 465-469. 